\title{
Autoantibodies in Breast Cancer Identify Proteins Involved in Self- Renewal and Epigenetic Chromatin Remodeling
}

\author{
Félix Fernández Madrid*,1-4, Wei Chen ${ }^{2,4}$, Naimei Tang ${ }^{1,4}$, Xinbo Zhang ${ }^{1,4}$, Jinsheng Dong ${ }^{1,4}$, \\ Harpreet Sagar ${ }^{1,4}$ and Judith Abrams ${ }^{1-4}$
}

\author{
${ }^{1}$ Department of Internal Medicine; ${ }^{2}$ Karmanos Cancer Institute; ${ }^{3}$ Center for Molecular Medicine and Genetics; ${ }^{4}$ Wayne \\ State University, Detroit, Michigan, USA
}

\begin{abstract}
Recent studies have shown that autoantibodies developing in cancer patients are tumor-associated and are promising biomarkers for the diagnosis and prognosis of cancer. Here we report a panel of signal transduction molecules with partial sequences identical to the oncogene Bmi-1, NIFK, a nucleolar protein interacting with the FHA domain of Ki67, TAB182, a protein interacting with tankyrase-1, CNOT6L, a subunit of the CCR4-NOT complex and to Elp3, a subunit of the elongator complex that are recognized as autoantigens by breast cancer sera with the ability to discriminate between invasive breast cancer and normal sera with high sensitivity and specificity. The proteins bearing the epitopes recognized by these antibodies have conserved regions involved in protein-protein interactions participating in regulatory processes such as self renewal, cell proliferation and survival, chromatin modulation, transcriptional silencing and organ patterning, usually ascribed to stem cell function. In this work we demonstrate by autoantigen microarray analysis that autoantibodies in breast cancer sera have the potential to delineate pathway connectivity. Our data indicate that several pathways involved in maintaining telomere stability are the target of an autoimmune reaction in breast cancer. These findings suggest that autoantibodies with the ability to recognize autoantigens involved in self-renewal and epigenetic chromatin remodeling have the potential to predict an invasive tendency of breast cancer.
\end{abstract}

Keywords: Autoantibodies, Bmi-1, autoantigen microarray, epigenetics, biomarkers, breast cancer.

\section{INTRODUCTION}

Recent studies have shown that antibodies to cancerassociated antigens appear early during carcinogenesis, that they are tumor relevant, and that they have potential as markers for the diagnosis and prognosis of cancer [1-8].

In our previous work on autoantibodies in the diagnosis of breast cancer [5,6] using a new antibody-based biomarker discovery approach [9], we reported a 12-phage breast cancer predictor group constructed with phage inserts recognized by sera from patients with breast cancer and not by non-cancer or autoimmune control sera with high sensitivity and specificity $[5,6]$. In that work we also identified several autoantigens including annexin XI-A, the KIAA1671 gene product, elongation factor-2, Grb2-associated protein 2, the p80 subunit of the Ku antigen, ribosomal protein S6, NIFK, a nucleolar protein interacting with the FHA domain of Ki67 and other unknown autoantigens that could significantly discriminate between breast cancer and non-cancer control sera and could distinguish ductal carcinoma in situ (DCIS) from invasive carcinoma (INV) of the breast [5,6].

In this work we have further characterized by sequence determination of phage displayed antigens, autoantigen microarray reactivity of breast cancer and control sera and

*Address correspondence to this author at the University Health Center, $4 \mathrm{H}$, Department of Internal Medicine, Division of Rheumatology, Wayne State University School of Medicine, 4201 St. Antoine Blvd., Detroit, MI 48201, USA; Tel: (313) 577-1133; Fax: 313-577-1938;

E-mail: fmadrid@med.wayne.edu homology searches, a small group of expression sequence tags (ESTs) cloned by biopanning a T7 cDNA library of breast cancer proteins with identity to the oncogene Bmi-1 and other proteins recognized as autoantigens by breast cancer sera. This is the first report of these autoantibodies in breast cancer. Since the proteins bearing the epitopes recognized by these autoantibodies have in common their ability to participate in regulatory processes such as self renewal and epigenetic chromatin remodeling, the aim of this study was to demonstrate the association of this autoantibody panel with the diagnosis of breast cancer, DCIS and INV of the breast. In this work we demonstrate by autoantigen microarray analysis that autoantibodies in breast cancer sera have the potential to delineate pathway connectivity and to contribute to the diagnosis of invasive breast cancer.

\section{MATERIALS AND METHODOLOGY}

\section{Selection of Sera for Immunoscreening and Biopanning of a T7 Phage cDNA Library}

Sera from women with breast cancer with comprehensive outcome data collected by the Karmanos Cancer Institute Breast Cancer Prognostic Study (KCI- BCPS) at Wayne State University from 1975 to 1985 , were used in this study. The characteristics of this cohort of patients have been reported [5]. The pathologic diagnoses of DCIS and INV of the breast in all patients were made when the tumor was first detected and sera from all women with breast cancer were obtained prior to any treatment. Nine sera from women with invasive breast carcinoma were chosen for immunoscreening a T7 phage cDNA display library of breast cancer proteins 
(Novagen), because they had strong, high titer IgG signals (1:500) on immunoblots, suggesting that the autoantibodies were abundant [5]. We screened plaques obtained from sequential rounds of immune-precipitation, and selected a subset of strongly recognized antigens to assemble a library of breast cancer autoantigens. After the final round of biopanning, phages were plated at low density on a lawn of E.coli, and plaque lifts were screened using patient sera to identify individual positive phages which were used to construct a breast autoantigen microarray as previously described [5].

\section{Autoantigen Microarray Construction, Hybridization, Quantification and Antigen Identification}

Plaque-pure phages were grown to high titer in bacterial cultures that were incubated until complete lysis. Supernatants collected after a 10 minute x $10,000 \mathrm{~g}$ spin were arrayed in 384-well microtiter dishes. The entire phage-library was spotted in duplicate onto nitrocellulose-coated FAST Slides (Schleicher and Schuell) using a Flexys robot (Genomic Systems). Each slide was probed either with a serum from patients with DCIS, INV of the breast or with a control serum and with a mouse monoclonal antibody directed against a non-variable $\mathrm{T} 7$ phage coat protein (Novagen, Madison, WI) [5].

Probing the T7 display cDNA library with sera from women with breast cancer and normal controls were performed as previously reported [5]. Sera used for immunoscreening the T7 cDNA library were not used in the hybridization step. Sera from 15 women with DCIS and from 22 women with INV carcinoma of the breast consecutively enrolled in the KCI- BCPS with biopsy-proven diagnoses were chosen to hybridize the breast autoantigen microarray. Thirty three non-cancer, non-autoimmune control sera were obtained from asymptomatic women having no past or family history of breast or ovarian cancer. All sera were stored frozen at $-80^{\circ} \mathrm{C}$ until use. Women chosen as controls were of similar age and race as the women with breast cancer (breast cancer mean age at diagnosis: 59.3 years; race, Caucasian, 95\%, African American, 5\%; control women, mean 56.4 years; race: Caucasian, 93\%, African American, 7\%). Patient reactivity was detected using CY3-labeled goat anti-human $\operatorname{IgG}+\operatorname{IgM}$ secondary antibodies, and the amount of phages present in each spot was measured by binding of CY5labeled goat anti-mouse $\operatorname{IgG}+\operatorname{IgM}$ antibodies. Phage slides also contained 96 "blank" spots (buffer only) to measure background, and 32 "control" spots, which consisted of identical phage clones spotted in 32 equidistant positions to assess positional variability across each slide. CY3 and CY5 signals were read using a GenePix 4000, a slide array reader (Axon Instruments), and quantified using ImaGene software
(ImaGene 5.1, BioDiscovery Inc). The CY3/CY5 ratios were calculated for each phage spot, and values for duplicate spots were averaged. We based the analysis on $\mathrm{CY} 3 / \mathrm{CY} 5$ ratios for all signals corresponding to cancer and control sera. Log2-transformation of the $\mathrm{Cy} 3 / \mathrm{Cy} 5$ ratios was applied [9]. For those ratios that were less and equal to 0 , a threshold of 0.1 was used. Quantile normalization was performed. The goal of this between array normalization was to impose the same empirical distribution of intensities to each array, so that the intensities from each array could be comparable. As some phages have very small variations across the arrays, only phages whose variations were in the top $25 \%$ were used in the analysis. The bootstrap "out-of-bag" validation method was used to estimate the distribution of the sensitivities and the specificities from the KNN classification. A sensitivity analysis of number of neighbors was also performed.

We determined the identity of the autoantigens that were significantly recognized by multiple breast cancer sera on the microarray by sequencing the phage cDNA inserts. After identifying informative phages, the corresponding cDNA inserts were amplified by PCR using primers flanking the insertion site, and sequenced by the core facility of the Center for Molecular Medicine and Genetics at WSU. After eliminating phage-related sequences, the GeneBank database was searched for sequence similarities to our identified cDNA sequences using the BLAST program [10]. This study had the approval of the Human Investigation Committee of WSU.

\section{RESULTS}

Autoantibodies Recognizing Antigens Involved in SelfRenewal and Epigenetic Chromatin Remodeling Distinguish INV from DCIS of the Breast and from Normal Individuals

We have reported that a panel of autoantibodies in breast cancer sera is able to distinguish breast cancer from normal sera with high sensitivity and specificity, while some antigens could distinguish INV from DCIS of the breast $[5,6]$. In subsequent work reported here, we have identified by immunoscreening a T7 phage cDNA library of breast cancer proteins and autoantigen microarray analysis a group of breast cancer autoantigens involved in self renewal and epigenetic mechanisms with potential to contribute to the early diagnosis of invasive carcinoma of the breast. Table 1 shows a group of expression sequence tags (ESTs) with identity to proteins involved in self renewal and epigenetic mechanisms which reacted with high titer IgG autoantibodies present in breast cancer sera [11-15].

Table 1. Breast Cancer Autoantigens Involved in Self-Renewal and Epigenetic Mechanisms

\begin{tabular}{|c|c|c|c|c|}
\hline Autoantigen & GenBank Account & Homology & BLASTn & tBLASTx \\
\hline \hline Bmi-1 [11] & $\underline{\text { CB331930 }}$ & Bmi-1 & $8 \mathrm{e}-38$ & $1 \mathrm{e}-9$ \\
\hline KIAA1671 [12] & $\underline{\text { CB331932 }}$ & TAB182 [13] & 0.0 & 116 \\
\hline RSAD1 [14] & $\underline{\text { CB334788 }}$ & Elp3 & $1 \mathrm{e}-115$ & $6 \mathrm{e}-48$ \\
\hline CNOT6L [15] & $\underline{\text { CB331931 }}$ & CNOT6L & 2e-75 & 5e-25 \\
\hline
\end{tabular}

The autoantigens identified by breast cancer sera by immunoscreening a cDNA library of breast cancer proteins, the account numbers of the cloned phage inserts (ESTs) in the GenBank, their homology revealed by searching databases and their corresponding e value using BLASTn and tBLASTx [10] are included. TB182, gi:116242814; RSAD1, radical Sadenosyl methionine containing domain, Elp3, NM_018346; CNOT6L, homo sapiens CCR4-NOT transcription complex, subunit 6-like, gi:115583678. 
The reported participation of the signal transduction molecules identified by immunoscreening in functions attributed to stem cells (Table 1) [16-21], prompted us to analyze the reactivity of a small probing sample of breast cancer sera with an autoantigen panel formed by Bmi-1 [11], CNOT6L[15], TAB182 [13], and RSAD1 [14] on a microarray of potential breast cancer autoantigens to assess for the association of these antigens with DCIS and INV of the breast.

Recently, Glinsky et al. identified Ki-67 as a component of an 11-gene signature associated with a conserved Bmi-1driven pathway suggested to represent a subset of highly malignant cancers with poor prognosis [22]. Since we have reported that autoantibodies to NIFK [23] [(CB331935)
MKI67, NM_032390] a nucleolar protein interacting with the forkhead associated (FHA) domain of Ki-67 [24] can contribute to identify sera from women with DCIS of the breast [5], we included this antigen in the analysis.

A side-by-side box-plot for each of the markers depicted in Table $\mathbf{1}$ and NIFK [23] is shown in Fig. (1).

The heat map shows the clustering of INV, DCIS and normal sera. While the Euclidean distance between INV of the breast and the other two groups is large, the distances between normal control individuals and DCIS of the breast are shorter. This analysis indicates that a panel constructed with these autoantigens has the potential of identifying sera from patients with invasive breast cancer.
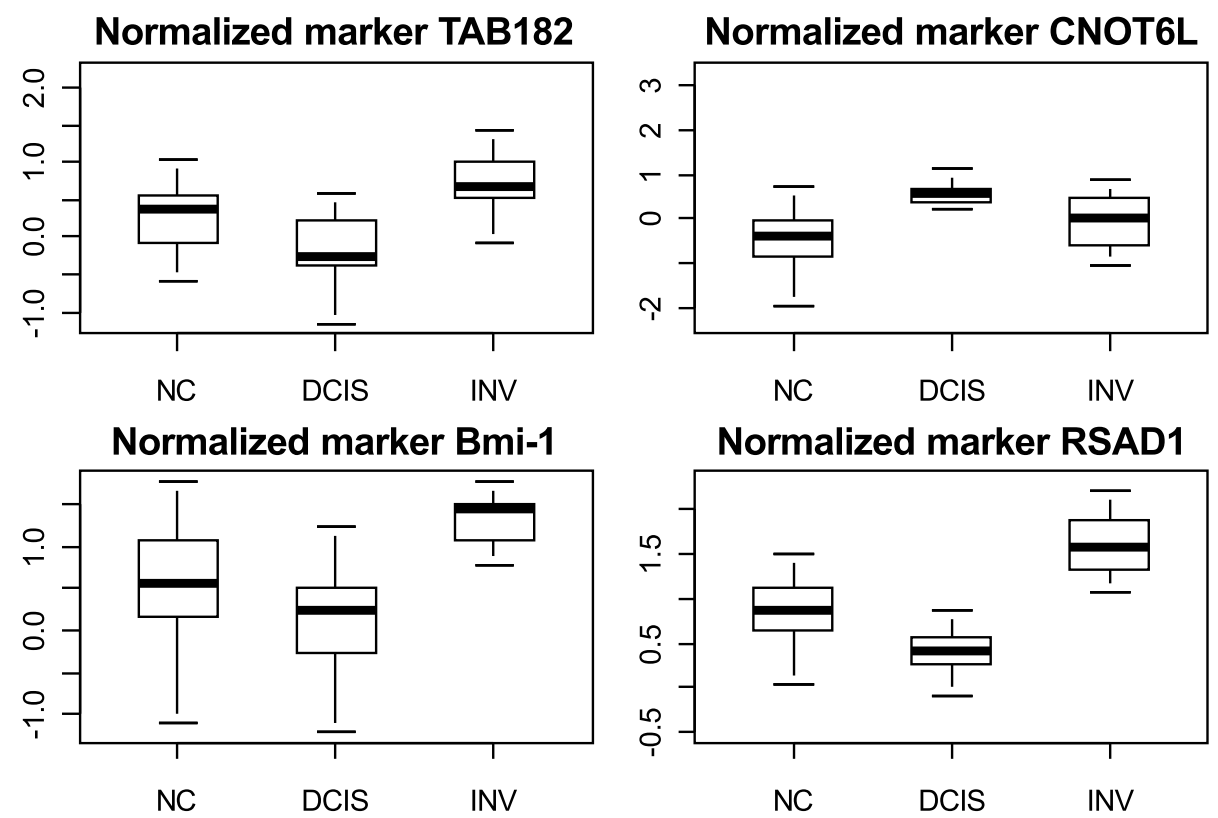

Normalized marker NIFK
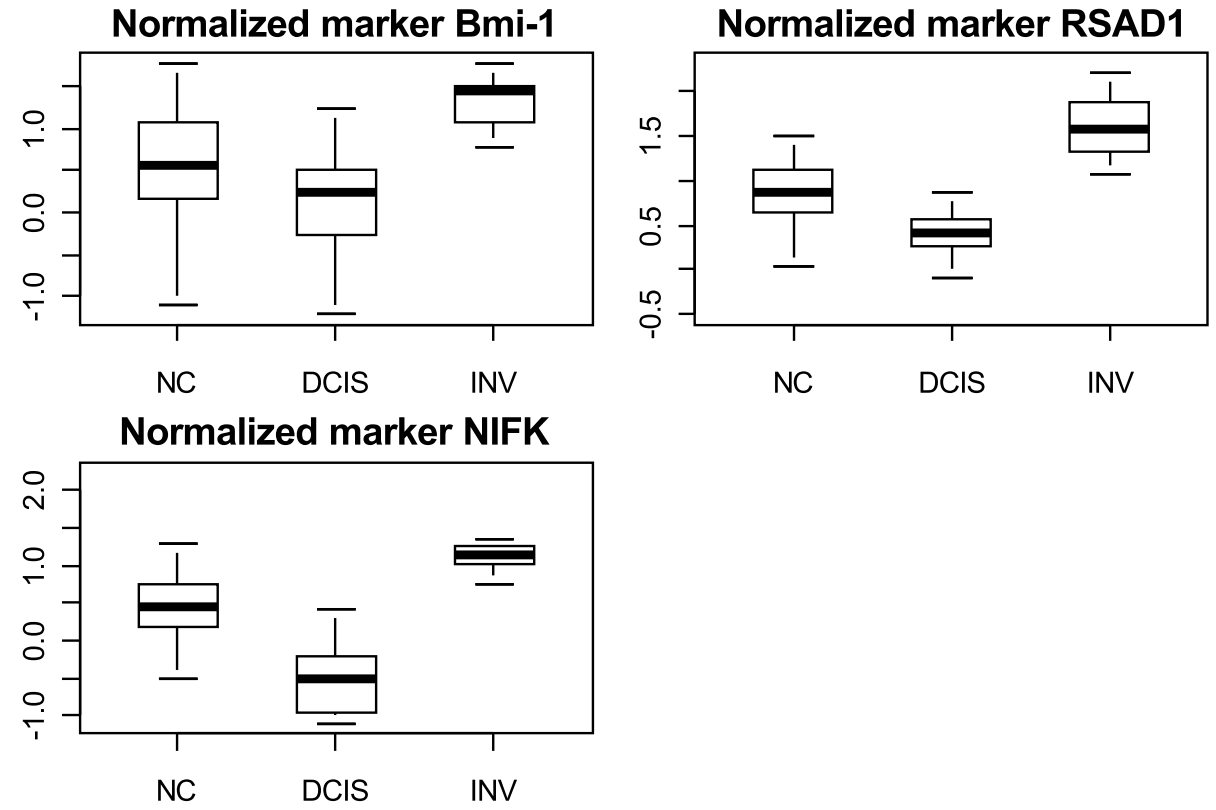

Fig. (1). Side-by-side box-plot of normalized expressions of 5 biomarkers potentially involved in self-renewal and epigenetic chromatin remodeling. Shows that the 5 markers were able to distinguish sera from patients with INV from those with DCIS of the breast or from normal control women. The $\mathrm{p}$ values from pair-wise Mann-Whitney tests are included in Table 2. The p values were adjusted using Hochberg's sharper Bonferroni procedure for strong control of the family wise error rate [25].

Table 2. Adjusted p Values from Mann-Whitney Tests

\begin{tabular}{|c|c|c|c|c|c|}
\hline & TAB182 & CNOT6L & Bmi-1 & RSAD1 & NIFK \\
\hline NC vs. DCIS & 0.018 & $<0.001$ & 0.168 & 0.002 & $<0.001$ \\
\hline NC vs. INV & 0.002 & 0.028 & $<0.001$ & $<0.001$ & $<0.001$ \\
\hline DCIS vs. INV & $<0.001$ & 0.028 & $<0.001$ & $<0.001$ & $<0.001$ \\
\hline
\end{tabular}

NC, normal non-cancer controls. All other abbreviations as in Table $\mathbf{1}$. 


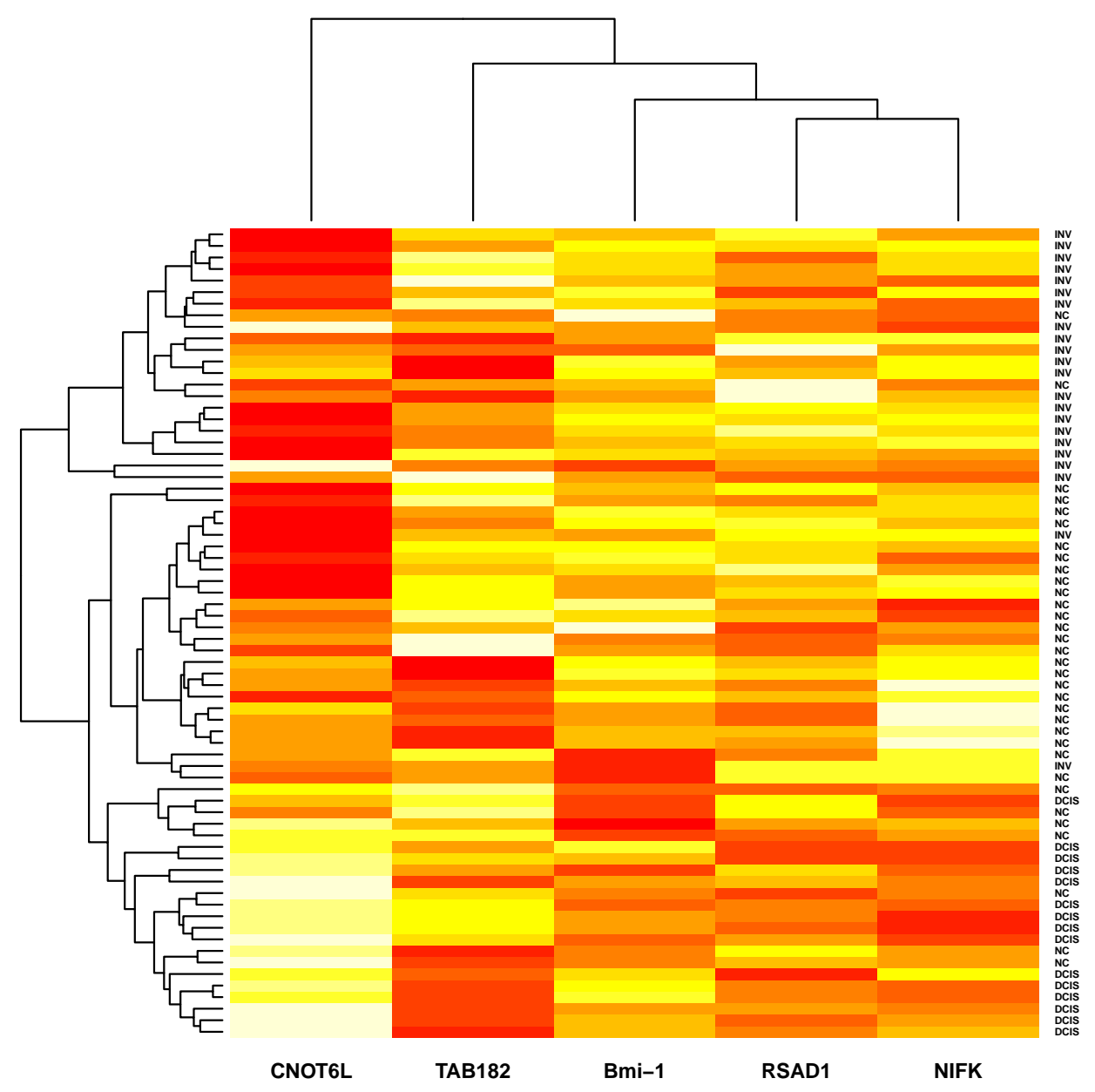

Fig. (2). A heat map depicts the performance of the 5 markers in the classification of two groups of breast cancer patients, DCIS and INV of the breast and normal control sera. To the right, clusters of INV, NC and DCIS of the breast. To the left, Euclidean distances between the clusters.

\section{K Nearest Neighbor Method}

The class of a test subject is determined by the majority vote of $\mathrm{k}$ nearest points using Euclidian distance in the training sample [26]. Before executing the KNN algorithm, each marker's $\log 2$ (ratio) was standardized across 70 samples. For the internal validation, a leave-one-out cross-validation approach was used to estimate the sensitivity and the specificity of this panel of biomarkers [27]. The operating characteristics of the knn $(k=5)$ yielded a sensitivity of 0.87 for DCIS and 0.82 for INV, while the specificity was 0.85 with an overall error of 0.16 .

\section{Some Pairs of Breast Cancer Autoantigens are Signifi- cantly Associated on the Microarray}

We have reported that autoantibodies to NIFK [23], a nucleolar protein interacting with the FHA domain of Ki-67 [24] can contribute to identify sera from women with DCIS of the breast $[5,6]$. Fig $(3)$ shows that autoantibodies to Bmi1 correlate with antibodies to NIFK and with those to RSAD1 and that antibodies to NIFK also correlate with the presence of antibodies to RSAD1 on the microarray (Fig. 3).

We have previously identified Grb2-AP2 [28] as a breast cancer autoantigen $[5,6]$. The same cloning serum led to the identification of both Grb2-AP2 and Bmi-1. Subsequent analysis of autoantigen reactivity on the microarray reported here, indicated that some breast cancer sera consistently recognize certain antigen pairs, in this case Bmi-1 and Grb2AP2. Since we have found that a cloning serum from women with breast cancer frequently identifies several antigens and likewise, that each probing serum from patients with breast cancer may react with more than one antigen on the microarray, we anticipated that analysis of the data may show a multiplicity (selection) effect resulting in an increased false positive significance rate. Thus, we attempted to determine whether the associations between pairs of antigens on the microarray are truly significant or whether they are due to a multiplicity effect, by controlling the false discovery rate of antigen association using the approach to multiple significance testing of Benjamini and Hochberg [29]. Using this method, the association between Grb2-AP2 and Bmi-1 on the microarray appeared to be significant $[\mathrm{p}=0.0005$; Benjamini-Hochberg $(\mathrm{B} / \mathrm{H})$ adjusted $\mathrm{p}=0.02]$.

\section{TAB182, a Tankyrase-Binding Protein and CNOT6L are Breast Cancer Autoantigens}

We have reported that partial sequences identical to the KIAA1671 protein [12] and to the ribosomal protein S6 [30] are breast cancer autoantigens $[5,6]$. Table 1 shows the results of a homology search indicating the identity of 

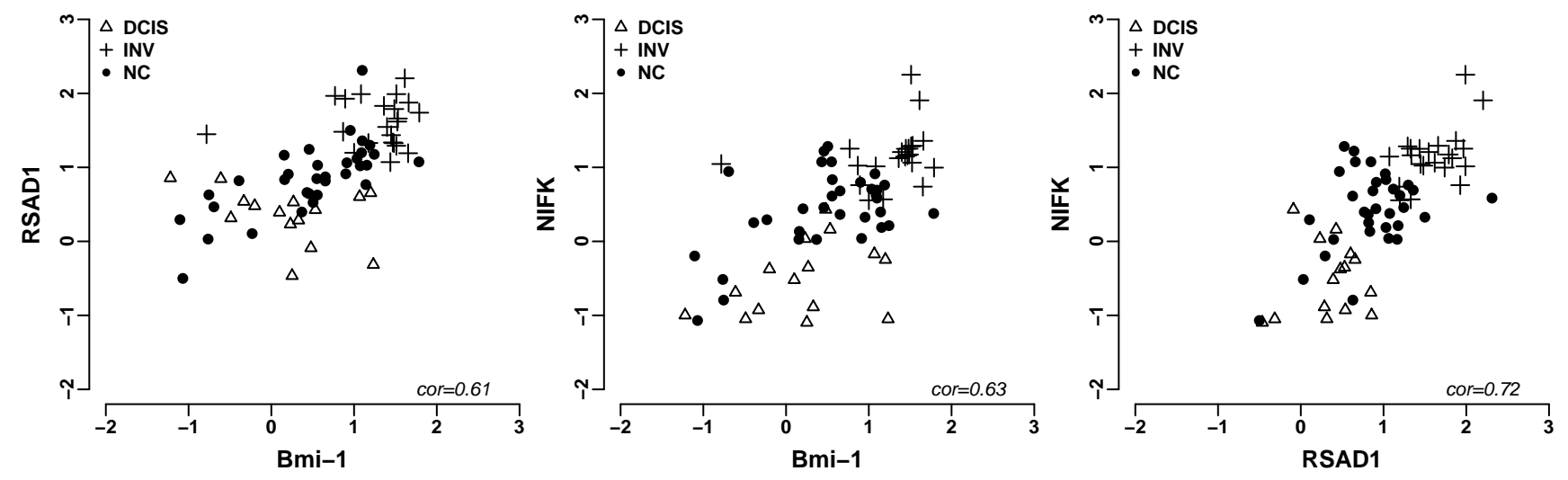

Fig. (3). Scatter plots of markers with correlation coefficient greater then 0.6.

KIAA1671 and TAB182, the tankyrase-1 binding protein [13]. This antigen was cloned by two different sera from patients with invasive carcinoma of the breast. By controlling the false discovery rate of antigen association using the approach to multiple significance testing [29] we found that TAB182 [13] was associated with the ribosomal protein S6 [30] on the autoantigen microarray $(p=0.00005$, and $B / H$ adjusted $\mathrm{p}=0.02$ ). We also report that the latter two antigens, were cloned using the same breast cancer serum. Homology searches demonstrated an association between TAB182 with Tankyrase 1 and 2, the CCR4-NOT transcription complex, subunit 6 [CNOT6L] as predicted functional partners [10]. In agreement with this prediction, we have identified CNOT6L, a subunit component of the CCR4-NOT and TAB182 as breast cancer autoantigens (Tables 1 and 2, Figs. 1 and 2). The CCR4-NOT complex is a highly conserved regulator of mRNA metabolism which is involved in protein-protein interactions. Its activation may reflect the involvement of the PI3/Akt kinase and MAPK kinases (ERK1/2 and p38) pathways as well as the co-regulatory activity of the IGF-I [15] (Fig. 4). It has been reported that depletion of endogenous CCR4-NOT subunits in breast cancer cells results in deregulation of ER alpha target genes [15]. Autoantibodies targeting the CCR4-NOT complex may reveal the activation of this pathway in certain patients with breast cancer.

We have reported that a partial sequence identical to $\mathrm{Ku} 80$ is recognized by multiple breast cancer sera and contribute to distinguish DCIS from INV of the breast $[5,6]$. $\mathrm{Ku} 80$ is an important component of the non-homologous end-joining (NHEJ) DNA repair pathway involved in the repair of DSBs in mammalian cells [31]. We have also shown that the $32 \mathrm{kDa}$ subunit of RPA is the target of autoantibodies in breast in breast cancer [3]. Other proteins which are commonly associated with DNA repair are also found at the telomere ends, such as Kup80, and tankyrase 1/2 [32]. DNA-PKcs as well as Kup80 are found at the ends of chromosomes, suggesting a further role for DNA-PKcs in the maintenance of telomere stability [33]. One of the roles of DNA-PKcs, alone or as part of a multi-protein complex, is to protect and to align broken ends of DNA [31]. P53, also a breast cancer autoantigen [34], is a substrate of DNA-PK $[35,36]$. Since there is increasing evidence implicating genomic instability in carcinogenesis [37], it is of interest that several signal transduction molecules involved in the NHEJ DNA repair pathway and in maintaining telomere stability are the target of an autoimmune reaction in breast cancer (Fig. 4).

\section{The Radical S-Adenosyl Methionine Domain of Elp-3 is Recognized by Breast Cancer Sera as an Autoantigen}

We have cloned a partial sequence identical to RSAD1 [14], radical S-adenosyl methionine containing domain 1 with breast cancer sera from two patients with invasive carcinoma of the breast (Table 1). This EST was recognized as an autoantigen by multiple breast cancer sera (Fig. 1) and its presence in breast cancer sera correlated with reactivity to Bmi-1 (Fig. 3). This sequence codes for elongator protein 3, MiaB family and includes a representative of the eukaryotic elongator subunit Elp-3.

\section{DISCUSSION}

The data show that the antibody-based approach used by us [9] allows to identify signal transduction molecules involved in pathway connectivity (Table 1, Figs. 3 and 4). Significant antibody reactivity recognizing an in frame EST with identity to an antigen on the microarray, indicates that such antigen is present in the cDNA library, and implies that the corresponding mRNA was being synthesized in the donor tumor and in the tumors from patients whose sera reacted on the microarray. We propose that pathway connectivity can be suggested by the recognition of signal transduction molecules in the cDNA T7 phage library by autoantibodies in cancer sera and by their association on the microarray. Potentially, this approach could provide information about pathway connectivity in individual patients.

The autoantibodies reported in this work recognize epitopes developing in proteins known to be involved in key molecular reactions participating in self renewal, cell differentiation, patterning, chromatin modifications and on the regulation of telomerase, processes thought to be involved in cancer stem cells functions [19-21]. Results from autoantigen microarray analysis suggest that these autoantibodies have the potential to discriminate between patients with invasive breast cancer and normal women with high sensitivity and specificity. The finding that the reactivity of 4 of the 5 the antibodies in women with DCIS of the breast was significantly lower than that of normal women was unexpected (Fig. 1 and Table 2). It is possible that the expression of these proteins is suppressed in women with DCIS and then 


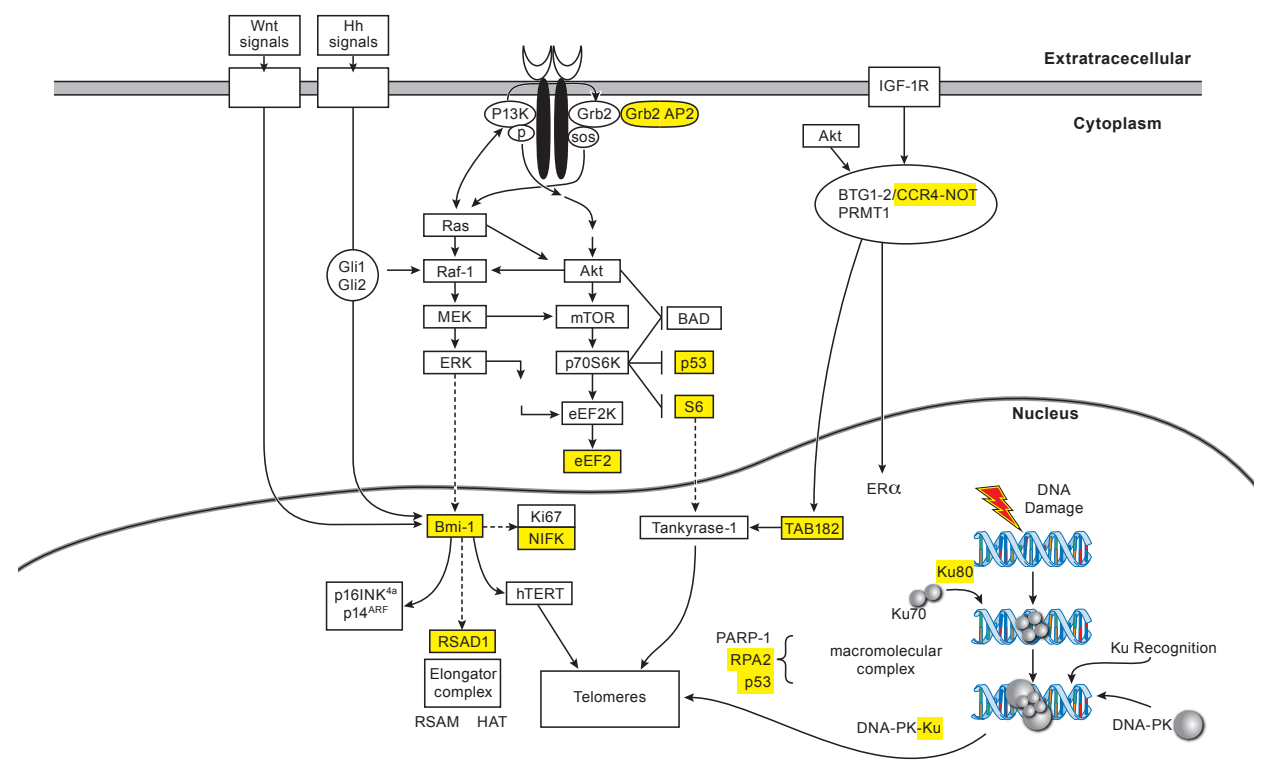

Fig. (4). Autoantibodies identify signal tranduction molecules that may be activated in breast cancer. Signal transduction molecules depicted in yellow elicit an autoimmune reaction in breast cancer. Interrupted arrows indicate possible pathway connectivity suggested by the autoantibody recognition of breast cancer-associated antigens. Abbreviations as in Table $\mathbf{1}$ and in the text.

rebounds in women with invasive cancer. It also possible that the molecular alterations which trigger an autoantibody response to the autoantigens reported here may be less prevalent in DCIS of the breast than in invasive carcinomas. Alternatively the observed effect may be due to unmeasured covariate differences between the groups of women.

From the group of phage-coded ESTs listed in Table 1, the Bmi-1 oncogene has attracted a great deal of interest because of its participation in functions attributable to stem cells $[11,17,18,38]$. Bmi-1 is a transcriptional repressor belonging to the polycomb group $(\mathrm{PcG})$ that plays a role in gene regulation in both normal and cancer stem cell proliferation through epigenetic mechanisms, such as methylation or acetylation of chromatin, initially reported as an oncogene collaborating with c-Myc in the induction of B-cell lymphomas [11]. The regulation of self-renewal of specific stem cells by several PcG genes, including Bmi-1, suggests a link between cell homeostasis and carcinogenesis $[39,40]$. The association of Bmi-1 overexpression with breast cancer has been reported [41,42] and it has been suggested that Bmi-1 regulates telomerase activity in MECs and plays a role in the development of human breast cancer [42].

The proliferation marker Ki-67 is widely used to detect proliferating cells, as it is only expressed during cell cycle progression [24]. Ki-67 contains an FHA domain and it has been suggested that this protein could interact with phosphorylated proteins through this domain [43]. Ki-67 is a component of an 11-gene signature associated with a conserved Bmi-1-driven pathway, suggested by Glinsky et al. to represent a subset of highly malignant cancers with poor prognosis, including lung, prostate and breast cancer [22]. Autoantibodies to Bmi-1 and to Ki-67 have also been reported in patients with lung cancer [44], consistent with the possibility that the presence of these antibodies may indicate an invasive tendency of malignancy. The Bmi-1-driven pathway reported by Glinsky et al, the association between the antibodies to Bmi-1 and NIFK in breast cancer sera (Fig.
3), and the finding that Bmi-1 and NIFK are recognized as breast cancer autoantigens by the sera of some women with breast cancer (Table 1) suggest that Bmi-1 may influence cell proliferation through Ki-67 (Fig. 4).

A number of developmental signaling pathways, such as Wnt, Notch, and Hedgehog ( $\mathrm{Hh})$, play a role in regulating the self-renewal of normal stem cells in the hematopoietic system, the skin, the nervous system, and the breast [18,21]. The effects of the Wnt and the Hh signaling pathways on growth and patterning during embryonic development are thought to be mediated by polycomb gene Bmi-1 [18,21]. It has also been demonstrated that Bmi-1 expression can be up-regulated by $\mathrm{Hh}$ signaling in human mammary stem/ progenitor cells [18]. We have reported that the Grb-2 AP2, the binding partner of Grb-2 [28] is a breast cancer autoantigen [5]. Effector molecules bind to phosphorylated tyrosine residues on the RTK's via the adaptor protein, Grb2/SOS [28]. Our finding that the same cloning serum led to the to the identification of both Grb2-AP2 and Bmi-1, and the recognition of this pair of antigens by multiple breast cancer sera on the microarray suggest the possibility that in some patients with breast cancer, signals transduced through the ras signaling cascade may activate Bmi-1 (Fig. 4). Recently, Datta et al. examined the oncogenic potential of Bmi-1 in MCF10A cells [45]. Although Bmi-1 overexpression alone did not result in oncogenic transformation in MCF10A cells, Bmi-1 co-overexpression with activated $\mathrm{H}$-ras resulted in efficient transformation of MCF10A cells in vitro.

We have reported that the KIAA 1671 protein is a breast cancer autoantigen $[5,6]$. Table 1 shows the results of a homology search indicating the identity of this protein with TAB182, the tankyrase-1 binding protein [13]. We have reported that partial sequences identical with the KIAA1671/tankyrase 1 antigen and the ribosomal protein S6 were significantly associated with the diagnosis of breast cancer $[5,6]$ This association may suggest that signals proceeding from mTOR may reach the telomeres via ribosomal 
protein S6 (Fig. 4). Tankyrases are poly (ADP-ribose) polymerases that enhance telomerase access to telomeres. Autoantibodies to the tankyrase 1 binding protein TAB182 are likely to reflect telomere dysfunction in breast cancer. Multiple evidence suggest that telomere-associated events are relevant to stem cell function, to senescence and to carcinogenesis [46]. It is relevant that other molecules located at the telomere ends are also targeted by autoantibodies in breast cancer. We have reported that a partial sequence identical to Ku80 is recognized by multiple breast cancer sera and contributes to distinguish DCIS from invasive carcinoma of the breast [5,6]. Ku80 is an important component of the NHEJ DNA repair pathway involved in the repair of double strand breaks in mammalian cells [31]. We have shown that the $32 \mathrm{kDa}$ subunit of RPA is the target of autoantibodies in breast cancer [3]. The autoimmune response triggered by multiple components of the DNA repair machinery, by Bmi1 and by the tankyrase 1 binding protein TAB182 in breast cancer sera suggests the involvement of the telomeres in breast carcinogenesis (Fig. 4).

We have identified CNOT6L, a subunit component of the CCR4-NOT complex as a breast cancer autoantigen (Table 1). The CCR4-NOT complex is involved in protein-protein interactions and its activation may reflect the involvement of the PI3/Akt kinase and MAPK kinases (ERK1/2 and p38) pathways as well as the co-regulatory activity of the IGF-I (Fig. 4) [15]. It has been reported that depletion of endogenous CCR4-NOT subunits in breast cancer cells results in deregulation of ER alpha target genes [15]. Autoantibodies targeting the CCR4-NOT complex may indicate the activation of this pathway in certain patients with breast cancer.

The identification of the RSAD1 domain of Elp-3 as a breast cancer autoantigen (Table 1) is another example of the participation of antibody-recognized epitopes involved in protein-protein interactions, instrumental in epigenetic chromatin remodeling. Identical sequences have been cloned from colon carcinoma, chondrosarcoma, islets of Langerhans, lung cancer as well as from embryonic tissues [14]. We speculate that the association of Bmi-1 and RSAD1 on the autoantigen microarray (Fig. 3) could be a clue indicating that Bmi-1 might influence the set of reactions catalised by RSAD1 (Fig. 4). Purification of chromatin-associated hyperphophorylated RNAPII from yeast led to the isolation of the elongator multisubunit complex [47]. The histone acetyltransferases and methyltransferases are part of a class of chromatin remodeling complexes that catalyze posttranslational histone acetylation and methylation reactions [48]. The Elp3 subunit of the elongator complex in humans contains a well characterized highly conserved C-terminal acetyltransferase (HAT) domain and its central region shares significant sequence homology to the radical SAM superfamily [49,50]. Human holo-elongator complex has robust histone acetyltransferase activity directed against histone H3 and $\mathrm{H} 4$ [50].

Whether the cells harboring the molecular changes responsible for eliciting an autoimmune reaction in breast cancer sera reported here, conform to the current definition of cancer stem cells or progenitor cells is unknown. A prospective validation study using high throughput microarray analyses could establish the value of these autoantibodies for the early diagnosis of invasive breast cancer. The identifica- tion of patients with tumors with invasive tendency at the time of diagnosis could have important implications for the choice of therapy of patients with newly diagnosed breast cancer.

The finding that some pairs of breast cancer autoantigens are associated on the microarray is reminiscent of the linked antibodies characteristic of systemic lupus erythematosus [51]. Although the significance of these findings is unknown, their presence in cancer sera merits further investigation.

The cancer stem cell hypothesis [19-21] has important implications in the field of biomarker discovery. Since the non-tumorigenic cells forming the bulk of the tumor are probably many cell cycles removed from the cells responsible for the cancer-initiating events and are thus heterogeneous and antigen diverse, a large portion of the tumorassociated antigens identified by currently used methods, although indeed tumor-associated, have probably originated in the bulk of the non-tumorigenic but yet antigenic cells. It is likely that a biomarker discovery approach targeting the cancer stem cell compartment may in the future, yield diagnostic and prognostic panels with the highest accuracy.

\section{CONCLUSION}

In summary, this is the first report of autoantibodies to Bmi-1 and other autoantibodies recognizing epitopes developing in proteins known to be involved in key molecular reactions participating in self renewal, cell differentiation, patterning, chromatin modifications and on the regulation of telomerase. A prospective study involving a larger number of patients and non-cancer controls should determine whether these autoantibodies can discriminate between patients with early forms of invasive breast cancer and normal women with high accuracy.

\section{ACKNOWLEDGEMENTS}

This work was supported by NIH grants R21 AR-99-128 (CA-87759) and R01 CA 122277-01A2 (PI: F Fernandez Madrid). We acknowledge the able contribution of Matthew Garin in the construction of figures.

\section{REFERENCES}

[1] Madrid F, VandeVord PJ, Yang X, et al. Antinuclear antibodies as potential markers of lung cancer. Clin Cancer Res 1999; 5: 1393400 .

[2] Tan E. Autoantibodies as reporters identifying aberrant cellular mechanisms in tumorigenesis. J Clin Investig 2001; 108: 1411-5.

[3] Tomkiel JE, Alansari H, Tang N, et al. Autoimmunity to the Mr 32,000 subunit of replication protein a in breast cancer. Clin Cancer Res 2002; 8: 752-8.

[4] Koziol JA, Zhang JY, Casiano CA, et al. Recursive partitioning as an approach to selection of immune markers for tumor diagnosis. Clin Cancer Res 2003; 9: 5120-6.

[5] Fernández Madrid F, Tang N, Alansari H, et al. Autoantibodies to annexin XI-A and other autoantigens in the diagnosis of breast cancer. Cancer Res 2004; 64: 5089-96.

[6] Fernández Madrid F. Autoantibodies in breast cancer sera: candidate biomarkers and reporters of tumorigenesis. Cancer Lett 2005; 230: 187-98.

[7] Wang X, Yu J, Sreekumar A, et al. Autoantibody signatures in prostate cancer. New Engl J Med 2005; 353: 1224-35.

[8] Chatterjee M, Mohapatra S, Donan A, et al. Diagnostic markers of ovarian cancer by high throughput antigen cloning detection on arrays. Cancer Res 2006; 66: 1181-90.

[9] Fernández Madrid F, Tang N, Alansari H, Karvonen RL, Tomkiel JE. Improved approach to identify cancer-associated autoantigens. Autoimmun Rev 2005; 4: 230-5. 
[10] Altschul SF, Madden TL, Schäffer AA, et al. Gapped BLAST and PSI-BLAST: a new generation of protein database search programs. Nucleic Acids Res 1997; 25: 3389-402.

[11] Haupt Y, Alexander WS, Barri G, Klinken S, Adams J. Novel zinc finger gene implicated as myc collaborator by retrovirally accelerated lymphomagenesis in E $\mu$-myc transgenic mice. Cell 1991; 65: 753-763.

[12] Hirosawa M, Nagase T, Murahashi Y, Kikuno R. Identification of novel transcribed sequences on human chromosome 22 by expressed sequence tag mapping. DNA Res 2001; 8: 1-9.

[13] Seimiya H, Smith S. The telomeric poly (ADP-ribose) polymerase, tankyrase 1, contains multiple binding sites for telomeric repeat binding factor 1 (TRF1) and a novel acceptor, 182-kDa tankyrasebinding protein (TAB182). J Biol Chem 2002; 277: 14116-26.

[14] Strausberg RL, Feingold EA, Grouse LH, et al. Generation and initial analysis of more than 15,000 full-length human and mouse cDNA sequences. Proc Natl Acad Sci USA 2002; 99: 16899-903.

[15] Winkler GS, Mulder KW, Bardwell VJ, Kalkhoven E, Timmers HT. Human Ccr4-NOT complex is a ligand-dependent repressor of nuclear receptor-mediated transcription. EMBO J 2006; 25: 308999.

[16] Park I-K, Qian D, Kiel M, et al. Bmi-1 is required for maintenance of adult self-renewing haematopoietic stem cells. Nature 2003; 423: 302-5.

[17] Lessard J, Sauvageau G. Bmi-1 determines the proliferative capacity of normal and leukemic stem cells. Nature 2003; 423: 255-60

[18] Liu S, Dontu G, Mantle ID, et al. Hedgehog signaling and Bmi-1 regulate self-renewal of normal and malignant human mammary gland stem cells. Cancer Res 2006; 66: 6063-71.

[19] Lapidot T, Sirard C, Vormoor J, et al. A cell initiating human acute myeloid leukemia after transplantation into SCID mice. Nature 1994; 367: 645-8.

[20] Sell S, Pierce GB. Maturation arrest of stem cell differentiation is a common pathway for the cellular origin of teratocarcinomas and epithelial cancers. Lab Investig 1994; 70: 6-22.

[21] Wicha MS, Liu S, Dontu G. Cancer stem cells: an old idea-a paradigm shift. Cancer Res 2006; 66: 383-90.

[22] Glinsky GV, Berezovska O, Glinskii AB. Microarray analysis identifies a death-from-cancer signature predicting therapy failure in patients with multiple types of cancer. J Clin Investig 2005; 115:1503-21.

[23] Tagaki M, Sueishi M, Saiwaki T. A novel nucleolar protein, NIFK, interacts with the forkhead associated domain of pKi-67. J Biol Chem 2001; 276: 25386-91.

[24] Endl E, Gerdes J. The Ki-67 protein: fascinating forms and an unknown function. Exp Cell Res 2000; 257: 231-237.

[25] Hochberg Y. A sharper Bonferroni procedure for multiple tests of significance. Biometrika 1988; 75: 800-802

[26] Hastie T, Tibshirani R, Friedman JH. The Elements of Statistical Learning: Data Mining, Inference, and Prediction. New York: Springer-Verlag 2001

[27] Yang YH, Paquet AC. Preprocessing Two-Color Spotted Arrays. Gentleman VJ, Carey W, Huber RA, Irizarry S, Dudoit S, Eds. Bioinformatics and computational biology solutions using $\mathrm{R}$ and bioconductor. New York: Springer-Verlag 2005.

[28] García-Echeverría C. Antagonists of the Src homology 2 (SH2) domains of Grb2, Src, Lck, and ZAP-70. Curr Med Chem 2001; 8: 1589-604

[29] Benjamini J, Hochberg Y. Controlling the false discovery rate. A practical and powerful approach to multiple testing. J Royal Stat Soc Series B 1995; 57: 289-300.
[30] Heinze H, Arnold HH, Fischer D, et al. The primary structure of the human ribosomal protein S6 derived from a cDNA clone. J Biol Chem 1988; 263: 4139-44

[31] Collis SJ, De Weese TL, Jeggo P, Parker AR. The life and death of DNA-PK. Oncogene 2005; 24: 949-61.

[32] Blackburn EH. Telomeres and telomerase: their mechanisms of action and the effects of altering their functions. FEBS Lett 2005; 579: 859-862.

[33] Autexier C, Lue NF. The structure and function of telomerase reverse transcriptase. Annu Rev Biochem 2006; 75: 493-517.

[34] Crawford L, Pim DC, Bulbrook RD. Detection of antibodies against the cellular protein p53 in sera from patients with breast cancer. Int J Cancer 1982; 30: 403-8.

[35] Shieh S-Y, Ikeda M, Taya Y. DNA damage-induced phosphorylation of p53 alleviates inhibition by MDM2. Cell 1997; 325-34.

[36] Mayo LD, Turchi JJ, Berberich SJ. Mdm-2 phosphrylation by DNA-dependent protein kinase prevents interaction with p53. Cancer Res 1997; 57: 5013-16.

[37] Lengauer C, Kinzler KW, Vogelstein B. Genetic instabilities in human cancers. Nature 1998; 396: 643-9.

[38] Buchwald G, van der Stoop P, Weichenrieder O, Perrakis A, van Louizen M, Sixma TK. Structure and E3-ligase activity of the Ring-Ring complex of Polycomb proteins Bmil and Ring $1 \mathrm{~b}$. EMBO J 2006; 25: 2465-74

[39] Valk-Linbeek ME, Bruggeman SW, van Louizen M. Stem cells and cancer; the polycomb connection. Cell 2004; 118: 409-18.

[40] Gil J, Bernard D, Peters G. Role of Polycomb group proteins in stem cell renewal and cancer. DNA Cell Biol 2005; 24: 117-25.

[41] Kim JH, Yoon SY, Jeong S-H, et al. Overexpression of Bmi-1 oncoprotein correlates with axillary lymph node metastases in invasive ductal breast cancer. Breast 2004; 13: 383-8.

[42] Dimri GP, Martinez JL, Jacobs JJL, et al. The Bmi-1 oncogene induces telomerase activity and immortalizes human mammary epithelial cells. Cancer Res 2002; 62: 4736-45.

[43] Li J, Lee GI, Van Doren SR, Walker JC. The FHA domain mediates phosphoprotein interactions. J Cell Sci 2000; 113: 4143-9.

[44] Zhong L, Hidalgo GE, Stromberg AJ, Hirschowitz EA. Identification of circulating antibodies to tumor-associated proteins for combined use as markers of non-small cell lung cancer. Proteomics. 2004; 4: 1216-25.

[45] Datta S, Hoenerhoff MJ, Bommi P, et al. Bmi-1 Cooperates with H-Ras to transform human mammary epithelial cells via dysregulation of multiple growth-regulatory pathways. Cancer Res 2007; 67: 10286-95.

[46] Sharpless NE, DePinho RA. Telomeres, stem cells, senescence, and cancer. J Clin Investig 2004; 113: 160-8.

[47] Otero G, Fellows J, Li Y, et al. Elongator, a multisubunit Component of a Novel RNA Polymerase II Holoenzyme for Transcriptional Elongation. J Mol Cell 1999; 3: 109-18.

[48] Chen J, Kinyamu HK, Archer TK. Changes in attitude, changes in latitude: nuclear receptors remodeling chromatin to regulate transcription. Mol Endocrinol 2005; 20: 1-13.

[49] Hawkes NA, Otero G, Winkler GS, et al. Purification and characterization of the human elongator complex. J Biol Chem 2002; 277 : 3047-52.

[50] Wittschieben BO, Otero G, de Bizemont T, et al. A novel histone acetyltransferase is an integral subunit of elongating RNA polymerase II holoenzyme. Mol Cell 1999; 4: 123-8.

[51] Tan EM. Antinuclear antibodies: diagnostic markers for autoimmune diseases and probes for cell biology. Adv Immunol 1989; 44: 93-151. 